\title{
Uterine Smooth Muscle Tumor of Uncertain Malignant Potential (STUMP): Case report
}

\author{
Ait Erraisse $\mathbf{M}^{1^{*}}$, Taghzout $\mathbf{H}^{1}$, El Fatemi $\mathbf{H}^{2}$, Belliraj $\mathrm{L}^{3}$, Lamrabet $\mathrm{FZ}^{4}$, Alami $\mathrm{B}^{5}$, El mazghi SA ${ }^{1}$, Bouhafa $\mathrm{T}^{1}$ and Hassouni $\mathrm{K}^{1}$ \\ ${ }^{1}$ Department of Radiation oncology, University hospital Hassan II Fez, Morocco \\ ${ }^{2}$ Department of Pathology, University hospital Hassan II Fez, Morocco \\ ${ }^{3}$ Department of Thoracic surgery, University hospital Hassan II Fez, Morocco \\ ${ }^{4}$ Department of Medical oncology, University hospital Hassan II Fez, Morocco \\ ${ }^{5}$ Department of Radiology, University hospital Hassan II Fez, Morocco \\ *Corresponding author: Ait Erraisse M, Department of Radiation oncology, university hospital Hassan II Fez, Morocco, Tel: +212 5356-19154, E-mail: \\ aiterraissemohamed@gmail.com
}

Received date: May 21, 2018, Accepted date: May 28, 2018, Published date: June 04, 2018

Copyright: (c) 2018 Ait Erraisse M, et al. This is an open-access article distributed under the terms of the Creative Commons Attribution License, which permits unrestricted use, distribution, and reproduction in any medium, provided the original author and source are credited.

\begin{abstract}
We report the case of a patient affected by a voluminous uterine tumor for which the histological analysis conclude in a smooth muscle tumor of uncertain malignant potential. This type of tumor is rare, but requires to be individualized to differentiate it from a benign hyperplasia or a sarcoma. The treatment must be made keeping in mind the risk of degeneration towards a malignant form.
\end{abstract}

Keywords : Uterus; STUMP; Smooth muscle

\section{Introduction}

Smooth muscle tumours of the uterus of uncertain malignant potential are a rare entity. Their diagnosis is histological. Their clinical presentation is similar to other tumours of the uterus. Imaging does not focus specifically on this diagnosis. Management is multidisciplinary and is based primarily on surgery. Radiation and chemotherapy have a place in degenerate forms. Hormone therapy is controversial. We report the case of a 50-year-old patient in whom, and after subtotal hysterectomy for postmenopausal bleedings for suspicion of myoma, histopathological examination found a smooth muscle uterine tumour with uncertain malignant potential or STUMP.

\section{Case study:}

This is a patient of 50 years, having been operated for postmenopausal bleedings for suspicion of uterine fibroma. She had an interannexial hysterectomy. On histopathological examination: tumor of the uterine wall, $5 \times 8 \mathrm{~cm}$, white, of firm consistency.

\section{On microscopy:}

The tumour is well limited, consisting of a proliferation of fusiform cells of the smooth muscle type (desmin, actin, and positive hormone receptors). Moderate to severe atypia are observed. The mitotic count is eight to nine mitoses for ten fields at high magnification. There is no tumour necrosis. the neck and the isthmus are not invaded. The diagnosis of a smooth muscle tumour with uncertain malignant potential has been made. No adjuvant treatment was proposed to the patient. CT follow up has been indicated. It showed 3 pulmonary lesions which stayed stable (Figure 1). 3 years later, there was an increase in size of the three lesions and a new limited paravertebral left parietal mass.

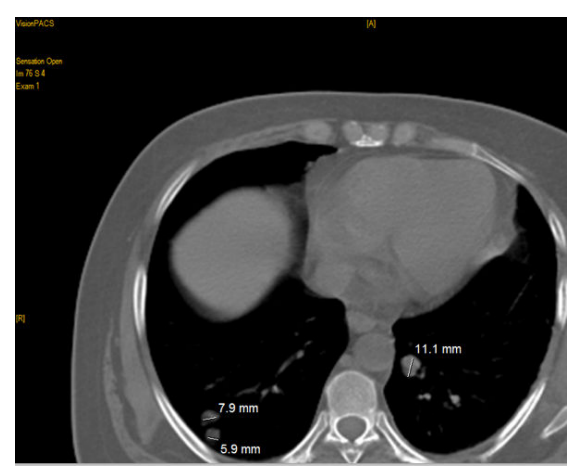

Figure 1: Axial CT slice showing three pulmonary lésions.

A wedge resection of the three pulmonary nodules showed two STUMP and one sarcoma on the third. A large excision of the parietal mass was performed and the pathological examination found a mass of $8 \times 9 \mathrm{~cm}$, well limited with the histology of a leiomyosarcoma with spindle cells expressing desmin and smooth muscle actin and not PS100. The resection was complete with a latissimus muscle free of any tumour proliferation. Adjuvant radiotherapy at a dose of $60 \mathrm{~Gy}$ was delivered to the paravertebral tumour bed and chemotherapy with doxorubicin with stability of the few pulmonary lesions appeared. A second line of gemcitabine-based chemotherapy was initiated with stabilization of the lesions. The follow up is for 5 years (Figure 2). 
Citation: $\quad$ Ait Erraisse M, Taghzout H, El Fatemi H, Belliraj L, Lamrabet FZ, et al. (2018) Uterine Smooth Muscle Tumor of Uncertain Malignant Potential (STUMP): Case report. J Nucl Med Radiat Ther 9: 365. doi:10.4172/2155-9619.1000365

Page 2 of 2

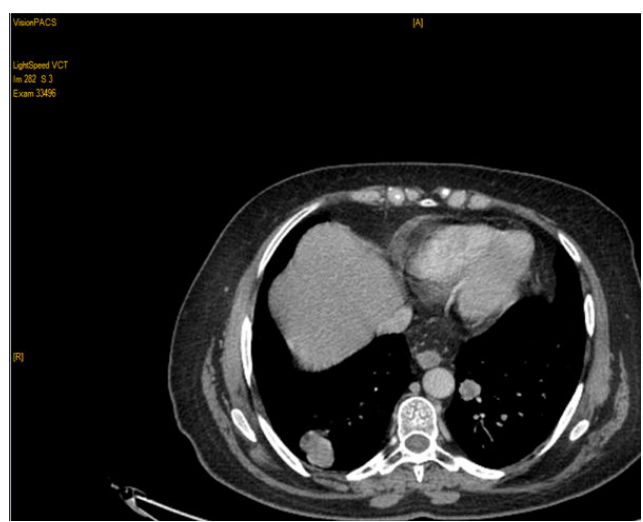

Figure 2: Axial CT slice showing an increase in size of the three previous lesions.

\section{Discussion}

Smooth muscle tumors of the uterus are subdivided into three categories: leiomyomas, leiomyosarcomas and STUMPs. The latter are smooth muscle tumors whose morphological characteristics do not allow to classify them properly in benign or malignant tumor (WHO). The histopronostic criteria for classifying smooth muscle tumors of the uterus are those proposed by Bell et al. in 1994 [1]. These criteria were validated by the WHO in 2003 [2]. These criteria are three and include nuclear atypia, the mitosis index and the presence or absence of tumor necrosis [3]. When one of the criteria is present and the second is difficult to evaluate, it is within the framework of the STUMP.

A fusiform cell smooth muscle tumor with moderate to severe diffuse nuclear atypia and a mitotic index difficult to assess or borderline between eight and nine mitoses in ten fields at 40X; a spindle cell smooth muscle tumor with moderate to severe nuclear atypia and necrosis whose tumor or ischemic nature is difficult to assess; a fusiform smooth muscle tumor with more than ten mitoses per ten 40X fields and a necrosis whose tumor or ischemic nature is difficult to assess; a true tumor necrosis in a "banal" leiomyoma (true tumor necrosis is the only criterion that can switch the diagnosis to a STUMP rather than a leiomyoma variant); STUMP also include uterine myxoid or epithelioid smooth muscle tumors with nuclear atypias, but with less than two to five mitoses per ten 40X fields [4].

The prognosis of STUMP is better than that of leiomyosarcomas (LMS). Peters et al. [5], studied 15 STUMP and 22 LMS and found 27\% relapse for STUMP versus 69\% for LMS with a five-year overall survival of $92 \%$ versus $40 \%$. In the context of LMS, local or distant recurrence is usually observed within two years of diagnosis. On the other hand, in STUMPs this recurrence can be much later. Berretta et al. [6], report a recurrence in the form of pulmonary metastasis nine years after a diagnosis of STUMP. Finally, the MD Anderson team reports its experience of 41 patients with the diagnosis of STUMP treated by myomectomy or hysterectomy, without additional treatment, and an average follow-up of 45 months. Three recurrences (7.3\%) are reported between 16 and 63 months, at the pelvic or pulmonary level, under a mode of STUMP (two cases) or leiomyosarcoma (one case), without any deaths [7].

\section{Conclusion}

STUMP is a very particular histopathological entity. Its clinical evolution is generally favourable. But atypical forms can exist and evolve rapidly to a malignant form including a leiomyosarcoma.The treatment is mostly surgical, but prospective studies must be performed to evaluate the benefit of adjuvant hormone therapy. The prognosis is rather favourable.

\section{References}

1. Bell SW, Kempson RL, Hendrickson MR (1994) Problematic ute- rine smooth muscle neoplasms: A clinicopathologic study of 213 cases. Am J Surg Pathol 18: 535-558.

2. Tavassoli FA, Devilee P (2003)World health organization classification of tumors: Tumors of the breast and female genital organs. Lyon: IARC Press, France.

3. Downes KA, Hart WR (1997) Bizarre leiomyomas of the uterus: A comprehensive pathologic study of 24 cases with long-term follow-up. Am J Surg Pathol 21: 1261-1270.

4. Prayson RA, Hart WR (1992) Mitotically active leiomyomas of the uterus. Am J Clin Pathol 97: 14-20.

5. Peters WA, Howard DR, Andersen WA, Figge DC (1994) Uterine smooth-muscle tumors of uncertain malignant potential. Obstet Gynecol 83: 1015-1020.

6. Berretta R, Rolla M, Merisio C, Giordano G, Nardelli GB (2008) Uterine smooth muscle tumor of uncertain malignant poten- tial: A three-case report. Int J Gynecol Cancer 18: 1121-1126.

7. Guntupalli SR, Ramirez PT, Anderson ML, Milam MR, Bodurka DC, et al. (2009) Uterine smooth muscle tumor of uncertain malignant potential: A retrospective analysis. Gynecol Oncol 113: 324-326. 